\title{
$r p o B$, a promising marker for analyzing the diversity of bacterial communities by amplicon sequencing
}

\author{
Jean-Claude Ogier ${ }^{1 *}$ D , Sylvie Pagès ${ }^{1}$, Maxime Galan ${ }^{3}$, Matthieu Barret ${ }^{2}$ and Sophie Gaudriault ${ }^{1}$
}

\begin{abstract}
Background: Microbiome composition is frequently studied by the amplification and high-throughput sequencing of specific molecular markers (metabarcoding). Various hypervariable regions of the 165 rRNA gene are classically used to estimate bacterial diversity, but other universal bacterial markers with a finer taxonomic resolution could be employed. We compared specificity and sensitivity between a portion of the rpoB gene and the V $3 \mathrm{~V} 4$ hypervariable region of the $16 \mathrm{~S}$ rRNA gene.

Results: We first designed universal primers for $r p o B$ suitable for use with Illumina sequencing-based technology and constructed a reference rpoB database of 45,000 sequences. The rpoB and V3 V4 markers were amplified and sequenced from (i) a mock community of 19 bacterial strains from both Gram-negative and Gram-positive lineages; (ii) bacterial assemblages associated with entomopathogenic nematodes. In metabarcoding analyses of mock communities with two analytical pipelines (FROGS and DADA2), the estimated diversity captured with the rpoB marker resembled the expected composition of these mock communities more closely than that captured with $\mathrm{V}_{3}$ V4. The rpoB marker had a higher level of taxonomic affiliation, a higher sensitivity (detection of all the species present in the mock communities), and a higher specificity (low rates of spurious OTU detection) than V3 V4. We compared the performance of the rpoB and V3 V4 markers in an animal ecosystem model, the infective juveniles of the entomopathogenic nematode Steinernema glaseri carrying the symbiotic bacteria Xenorhabdus poinarii. Both markers showed the bacterial community associated with this nematode to be of low diversity ( $<50$ OTUs), but only rpoB reliably detected the symbiotic bacterium $X$. poinarii.

Conclusions: Our results confirm that different microbiota composition data may be obtained with different markers. We found that rpoB was a highly appropriate marker for assessing the taxonomic structure of mock communities and the nematode microbiota. Further studies on other ecosystems should be considered to evaluate the universal usefulness of the rpoB marker. Our data highlight two crucial elements that should be taken into account to ensure more reliable and accurate descriptions of microbial diversity in high-throughput amplicon sequencing analyses: i) the need to include mock communities as controls; ii) the advantages of using a multigenic approach including at least one housekeeping gene ( $r p o B$ is a good candidate) and one variable region of the $16 \mathrm{~S}$ rRNA gene. This study will be useful to the growing scientific community describing bacterial communities by metabarcoding in diverse ecosystems.
\end{abstract}

Keywords: rpoB, $16 \mathrm{~S}$ rRNA gene, Metabarcoding, Mock communities, Entomopathogenic nematodes

\footnotetext{
* Correspondence: Jean-Claude.Ogier@umontpellier.fr

${ }^{1}$ DGIMI, INRA, University Montpellier, 34095 Montpellier, France

Full list of author information is available at the end of the article
}

(c) The Author(s). 2019 Open Access This article is distributed under the terms of the Creative Commons Attribution 4.0 International License (http://creativecommons.org/licenses/by/4.0/), which permits unrestricted use, distribution, and reproduction in any medium, provided you give appropriate credit to the original author(s) and the source, provide a link to the Creative Commons license, and indicate if changes were made. The Creative Commons Public Domain Dedication waiver (http://creativecommons.org/publicdomain/zero/1.0/) applies to the data made available in this article, unless otherwise stated. 


\section{Background}

The recent emergence of high-throughput sequencing platforms has revolutionized the study of complex microbial communities. Many of these studies involve the PCR amplification and sequencing of a taxonomic marker from complex communities of organisms. The sequences obtained can then be compared with databases of known sequences to identify the taxa present in the microbial community. The $16 \mathrm{~S}$ rRNA gene is the most common marker used for this purpose in bacterial ecology, particularly as exhaustive reference databases have been compiled for this taxonomic marker: the Greengenes database [1], the Ribosomal Database Project (RDP) [2], and SILVA [3], for example. In addition to their extensive catalogs of curated 16S rRNA gene sequences, each of those portals also offers a series of tools for sequence analysis. However, $16 \mathrm{~S}$ rRNA markers are also known to be the major source of bias in the amplicon sequencing approach [4]. Estimates of microbial diversity are generally biased by the variable number and sequence heterogeneities of 16S rRNA operons in bacterial species, generally leading to an overestimation of species richness [5, 6]. Furthermore, a growing number of publications have reported limitations to the use of the 16S rRNA gene in ecological studies due to its poor discriminatory power in certain bacterial genera [7], resulting in poor discrimination between species. In light of these major drawbacks, alternative, or at least complementary taxonomic markers should be sought for metabarcoding projects.

A few studies have tested other taxonomic markers by targeting conserved protein-coding genes, also known as housekeeping genes. Like the 16S rRNA gene, housekeeping genes are essential and ubiquitous genes universally present in the bacterial kingdom. However, these housekeeping genes evolve much more rapidly than the16S rRNA gene, and are therefore useful for differentiating between lineages that have recently diverged [8]. Moreover, these housekeeping genes are generally present as single copies in bacterial genomes, limiting the overestimation of operational taxonomical units (OTUs) in microbial assemblages. Despite these advantages, they are very rarely used in metabarcoding analyses, mostly due to the lack of an exhaustive sequence database for these genes. The protein-coding genes that have been tested for the assessment of microbial diversity include the genes for DNA gyrase subunit B (gyrB) ([8-10], RNA polymerase subunit B $(\operatorname{rpoB})[7,11,12]$, the TU elongation factor (tuf) [13], the $60 \mathrm{kDa}$ chaperonin protein (cpn60) [14].

The objective of this study was to assess the potential of the $r p o B$ gene as an alternative universal phylogenetic marker for metabarcoding analysis. The $r p o B$ gene has a number of potential advantages. Previous reports have shown this marker to be suitable for phylogenetic analyses, as it provides a better resolution at species level than the $16 \mathrm{~S}$ rRNA gene [15-18]. Moreover, the rpoB gene is sufficiently long $(4026 \mathrm{nc})$ for the rational identification of conserved genomic regions for the design of universal primers for the amplification of short barcodes (reads $<300 \mathrm{nc}$ ). Specific rpoB primer sets targeting Proteobacteria have been designed for high-throughput sequencing studies [12], but universal rров primers have not been tested in nextgeneration sequencing studies.

We designed universal rpoB primers suitable for use with Illumina sequencing-based technology and we constructed an $r p o B$ reference database of 45,000 sequences. The specificity and sensitivity of $r p o B$ were assessed on a mock bacterial community composed of DNA extracted from 19 strains, and compared with those for the V3 V4 regions of the $16 \mathrm{~S}$ rRNA gene. We also applied this community profiling approach to infective juveniles (IJs) of Steinernema glaseri, an entomopathogenic nematode [19, 20]. IJs are specifically associated with the intestinal bacterium Xenorhabdus poinarii (Enterobacteriaceae) [21, 22], which is therefore an effective control for evaluating the performance of taxonomic markers for Illumina sequencing analyses. We found that bacterial richness, in both mock communities and the nematode microbiota, was estimated more accurately with the $r p o B$ marker than with the $16 \mathrm{~S}$ rDNA marker, confirming the advantage of $r p o B$ as an alternative or complementary marker to the traditional variable regions of the 16S rRNA gene.

\section{Results}

Comparison of rpoB and V3 V4 markers distinguishing by sanger sequencing 19 individual taxa

We compared the taxonomic discrimination potentials of the $r p o B$ and V3 V4 markers, by extracting genomic DNA from the individual bacterial strains used to constitute the mock communities; then amplifying and Sanger sequencing the rpoB gene fragment ( $430 \mathrm{bp})$ and the $\mathrm{V} 3 \mathrm{~V} 4$ region of the 16S rRNA gene ( $\sim 50 \mathrm{bp})$. We used the RDP classifier tool (sequence similarity threshold $=97 \%$, bootstrap confidence cutoff $=80 \%$ ) to assign the sequences to taxa (Table 1). Taxonomic affiliation was determined more precisely with the rpoB gene fragment than with the V3 V4 region, because 13 and 0 OTUs were affiliated to a species with $r p o B$ and $\mathrm{V} 3 \mathrm{~V} 4$, respectively (Table 1 and Fig. $1 \mathrm{~A}$, lanes "Expected-rpo $B$ or Expected-16S"). Moreover, the rpoB marker had a higher sensitivity than the V3 V4 marker (19 OTUs versus 17 OTUs, Table 1).

\section{Comparison of the rpoB and V 3 V4 markers for metabarcoding descriptions of the bacterial community making up five artificial mock communities}

We constituted five different mock communities differing in the proportions of two taxa, Xenorhabdus 
Table 1 List of strains used for the extraction of the DNAs used to constitute the mock communities

\begin{tabular}{|c|c|c|c|c|}
\hline \multirow[b]{2}{*}{ Strain name } & \multirow[b]{2}{*}{ Phylum } & \multicolumn{3}{|c|}{ Taxonomic assignment based on Sanger sequencing of } \\
\hline & & The entire $16 \mathrm{~S}$ rRNA gene ${ }^{1}$ & The V3 V4 region ${ }^{2,3}$ & The rpoB region 2,3 \\
\hline $\mathrm{F} 1$ & $\gamma$-Proteobacteria & X. nematophila & Xenorhabdus & X. nematophila \\
\hline ТТ01 & & P. luminescens & Photorhabdus & P. luminescens \\
\hline TUR03-22 & & S. liquefaciens & Serratia & S. liquefaciens \\
\hline SW4 & & P. protegens & Pseudomonas & P. protegens \\
\hline SK39-ApoA & & P. chlororaphis & Pseudomonas & P. chlororaphis \\
\hline SW5 & & P. putida & Pseudomonas & P. putida \\
\hline B163 & & S. maltophilia & Stenotrophomonas & S. maltophilia \\
\hline FR211A & & Acinetobacter sp. & Acinetobacter & Acinetobacter \\
\hline SC & $\beta$-Proteobacteria & A. faecalis & Alcaligenes & A. faecalis \\
\hline SK39-ApoC & & Achromobacter & Achromobacter & Achromobacter \\
\hline MW8A4 & & Delftia sp. & Delftia & D. acidovorans \\
\hline BS3-2-1 & & Variovorax sp. & Variovorax & V. paradoxus \\
\hline 173 & & Acidovorax sp. & Acidovorax & Acidovorax sp \\
\hline Sphingo & á-Proteobacteria & Sphingomonas sp. & Sphingomonas & Sphingomonas \\
\hline TCH07-Apo2-4 & & Brevundimonas sp. & Brevundimonas & B. diminuta \\
\hline SW2 & & O. anthropi & Brucellacae & O. anthropi \\
\hline FR211C & Bacteroidetes & Sphingobacterium sp. & Sphingobacterium & Sphingobacterium \\
\hline SK72-2 & Fimicutes & Paenibacillus sp. & Paenibacillus & Paenibacillus \\
\hline SG4 & & E. mundtii & Enterococcus & E. mundtii \\
\hline
\end{tabular}

${ }^{1}$ based on NCBI Blast taxonomic assignment

${ }^{2}$ based on RDP Classifier assignment (bootstrap confidence $=0.9$ )

${ }^{3}$ gene fragments used for metabarcoding analyses

nematophila and Photorhabdus luminescens. The proportions of the 19 bacterial species making up the five mock communities, in terms of genome equivalents, are shown in Table 2. We performed metabarcoding analyses of the five mock communities (three technical replicates per mock community) with both $r p o B$ and the V3 V4 region of the $16 \mathrm{~S}$ rRNA gene (referred to hereafter as 16S). The targets were amplified and sequenced, and the sequences were processed with two different pipelines, FROGS (Find Rapidly OTUs with Galaxy Solution) and DADA2, to minimize bias related to the sequence analysis process. For the rрoB region, we obtained 224,129 reads (mean of 14,941 reads per sample) and 251,963 reads (mean of 16,797 reads per sample) after FROGS and DADA2 processing, respectively (Additional file 1 ). For the $16 \mathrm{~S}$ marker, we obtained 266,494 reads (mean of 17,766 reads per sample) and 206,641 reads (mean of 13,776 reads per sample) after FROGS and DADA2 processing, respectively (Additional file 1). Rarefaction curves obtained with quality-filtered reads indicated that sequencing depth was sufficient for both $16 \mathrm{~S}$ and $r p o B$ (Additional file 2). A comparison of the taxonomic affiliation levels of the observed OTUs (Fig. 1A) confirmed that the $r p o B$ gene fragment had the higher taxonomic discriminating potential; 60 to $80 \%$ of OTU assignations were at the species level for rрoB marker, but only $20 \%$ for the $16 \mathrm{~S}$ marker (Fig. $1 \mathrm{~A}$ ). We also noted that numerous OTUs (30 to $40 \%$ ) could not be assigned to a finer taxonomic level than the family with the $16 \mathrm{~S}$ marker.

For each sample mock community replicate, OTU numbers after the application of two thresholds to individual sample read abundances $(0.1$ and $1 \%)$ are indicated in Additional file 1. We evaluated the impact of the use of the rpoB and $16 \mathrm{~S}$ markers on the number of OTUs detected in metabarcoding analyses (Fig. 2). The numbers of OTUs detected depended on the marker, and were generally greater for the $16 \mathrm{~S}$ than for the rpoB marker; they also depended on the analysis pipeline. Interestingly, depending on the abundance threshold used, both markers either overestimated $($ cutoff $=0.1 \%)$ or underestimated $($ cutoff $=$ $1 \%)$ the number of OTUs.

We refined the comparison between the two markers, by aggregating and aligning the nucleotide sequences obtained by Illumina-sequencing of the three replicates for each mock community and building phylogenetic trees with the maximum likelihood method. The results 
Table 2 Composition of the five mock communities

\begin{tabular}{|c|c|c|c|c|c|}
\hline Bacterial species name & $\operatorname{Mock} 1^{a}$ & $\operatorname{Mock}^{a}$ & Mock3 $^{a}$ & Mock4$^{a}$ & Mock5 $^{a}$ \\
\hline Enterococcus mundtii & 0.09 & 0.01 & 0.17 & 0.17 & 0.17 \\
\hline Alcaligenes faecalis & 1.58 & 0.11 & 2.81 & 2.91 & 2.93 \\
\hline Variovorax paradoxus & 2.29 & 0.16 & 4.08 & 4.23 & 4.25 \\
\hline Pseudomonas protegens & 2.07 & 0.14 & 3.69 & 3.83 & 3.85 \\
\hline Stenotrophomonas maltophilia & 8.39 & 0.57 & 14.93 & 15.50 & 15.57 \\
\hline Brevundimonas diminuta & 6.15 & 0.42 & 10.94 & 11.36 & 11.41 \\
\hline Serratia liquefaciens & 1.45 & 0.10 & 2.58 & 2.68 & 2.69 \\
\hline Acinetobacter sp. & 6.21 & 0.42 & 11.05 & 11.47 & 11.52 \\
\hline Pseudomonas chlororaphis & 3.10 & 0.21 & 5.51 & 5.72 & 5.74 \\
\hline Ochrobactrum anthropi & 2.15 & 0.15 & 3.83 & 3.98 & 4.00 \\
\hline Pseudomonas putida & 3.11 & 0.21 & 5.53 & 5.74 & 5.77 \\
\hline Sphingobacterium sp. & 5.72 & 0.39 & 10.17 & 10.56 & 10.60 \\
\hline Acidovorax sp. & 2.24 & 0.15 & 3.98 & 4.14 & 4.15 \\
\hline Achromobacter sp. & 1.42 & 0.10 & 2.53 & 2.63 & 2.64 \\
\hline Paenibacillus sp. & 3.21 & 0.22 & 5.72 & 5.93 & 5.96 \\
\hline Delftia sp. & 1.41 & 0.10 & 2.51 & 2.61 & 2.62 \\
\hline Sphingomonas sp. & 3.28 & 0.22 & 5.83 & 6.05 & 6.08 \\
\hline Xenorhabdus nematophila & 35.87 & 36.69 & 3.35 & 0.40 & 0.04 \\
\hline Photorhabdus luminescens & 10.24 & 59.64 & 0.80 & 0.08 & 0.01 \\
\hline
\end{tabular}

${ }^{a}$ percentage based on genome number equivalents

for the mock 1 community analysis are shown (see Fig. 3 and Additional file 3 for $r p o B$ marker; Fig. 4 and Additional file 4 for $16 \mathrm{~S}$ marker). As a control, we built a phylogenetic tree with the sequences obtained by Sanger sequencing of both the rpoB and 16S markers after PCR amplification from the DNA of each separate bacterial species (Fig. 3A and $4 \mathrm{~A}$ ). With the $0.1 \%$ cutoff, a comparison with the topology of the Expected_rpoB and Expected_16S mock communities showed aberrant clusters in Illumina-sequenced mock communities (in gray in Figs. 3 and 4). We removed these clusters, which probably corresponded to chimeric sequences that had escaped the filtering process. With the rpoB marker, we were able to identify the 19 bacterial taxa of the mock1 community with perfect-match taxonomic identities (sensitivity $=100 \%$ ), but we noted the presence of a few sequence variants (12 and 4 sequences variants in the FROGS and DADA2 analyses, respectively). For the $16 \mathrm{~S}$ rRNA gene marker, the sensitivity for OTU detection was $100 \%$ for FROGS and $76 \%$ for DADA2, but we observed many more sequence variants than with the $r p o B$ marker (24 and 25 sequence variants with FROGS and DADA2, respectively), potentially due to $\mathrm{PCR} /$ sequencing errors or intragenomic polymorphisms for $16 \mathrm{~S}$ rRNA copy number. With the $1 \%$ cutoff, no chimeric sequences or sequence variants were detected with either the rpoB or the 16S rRNA gene marker, but the OTU detection sensitivity decreased considerably (between 58 and 64\%) (Figs. 3 and 4 and Additional files 3 and 4). We therefore selected the $0.1 \%$ cutoff as yielding the optimal sensitivity for OTU detection, despite the slight overestimation observed.

\section{Assessment of the quantitative potential of the rpoB and $16 \mathrm{~S}$ markers for metabarcoding approaches}

For visualization of the potential abundance biases during metabarcoding, we generated boxplots of the relative abundances of the 19 taxa making up the mock communities (as shown in Fig. 5 for the Mock3 community and in Additional file 5 for the other mock communities, except for mock2, for which the highest abundance of Xenorhabdus and Photorhabdus masked the presence of other bacteria). For both rpoB and $16 \mathrm{~S}$ markers, we observed differences in the relative abundances of OTUs between results from metabarcoding analysis and the expected OTU composition. For the five mock communities, we calculated the abundance bias ratios for each of the 19 taxa (Table 3). Interestingly, these ratios were not correlated with a particular phylogenetic group, and instead depended on both the bacterial strains and markers used for metabarcoding. These results confirm that, even with a single-copy gene such as $r p o B$, the measurement of OTU relative abundance is not strictly reliable, and metabarcoding should therefore be considered a semi-quantitative method. 


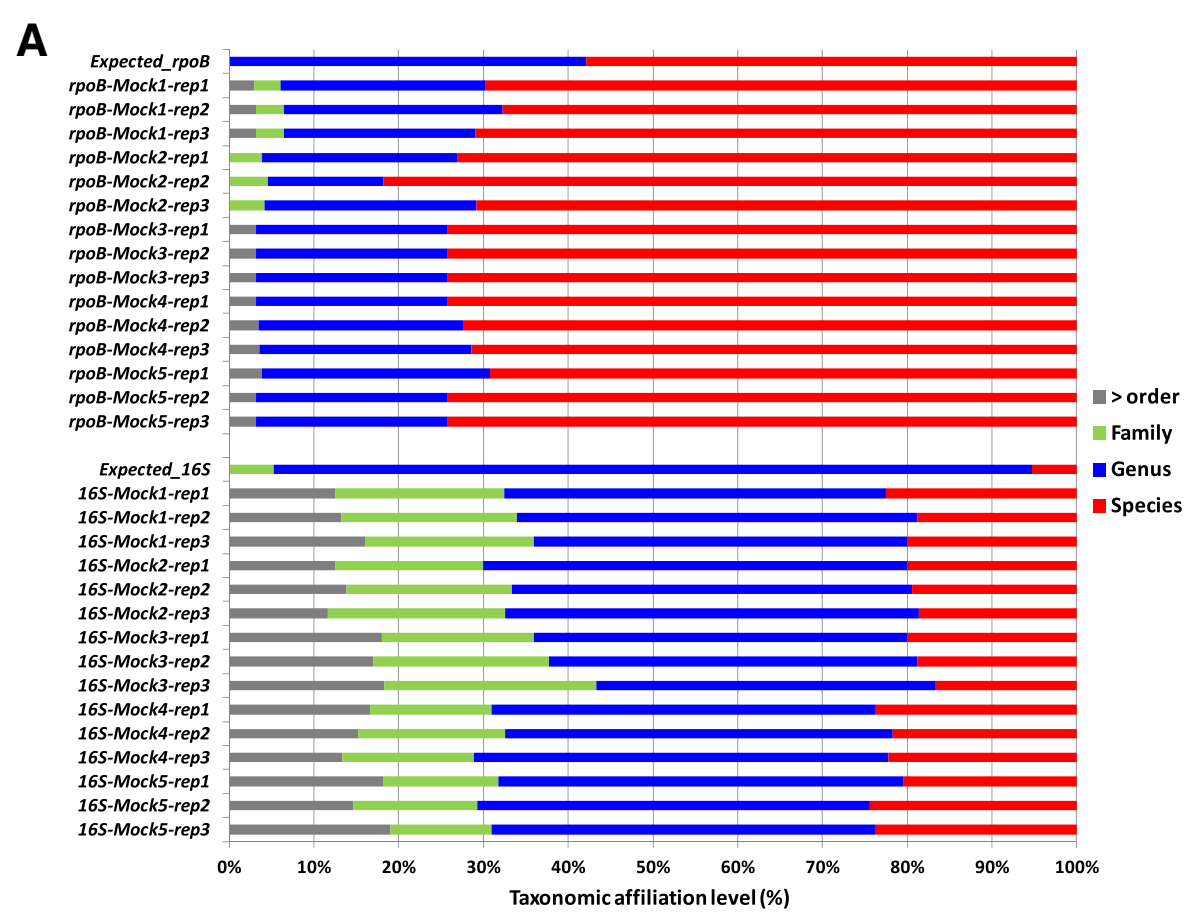

B

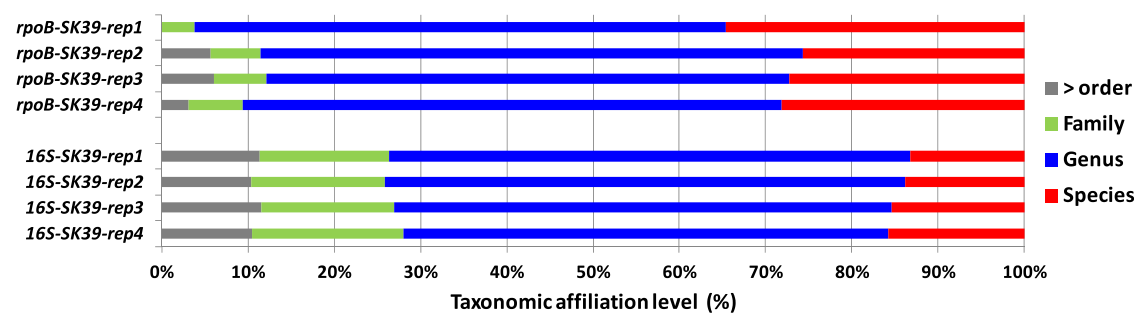

Fig. 1 Impact of the metabarcode markers on OTU taxonomic affiliations. Histogram showing the percentage of OTU affiliations at different taxonomic ranks ( $x$-axis) for $r p o B$ and 165 Illumina-amplicon sequencing for (a) the 15 mock community samples (five mock communities and 3 replicates per mock community) (b) the microbiota of the nematode Steinernema glaseri SK39 (four replicates). Gray, green, blue and red histogram bars correspond to the taxonomic ranks of order (and higher levels), family, genus and species, respectively. The total number of OTUs observed after sequence analysis via the FROGS pipeline was included in the analysis. The Expected_rpoB and Expected_16S lanes correspond to the taxonomic affiliations determined by Sanger sequencing of each of the taxa making up the mock communities

\section{Efficiency of the $r p o B$ marker for describing the microbiota of entomopathogenic nematodes}

We performed metabarcoding analyses on a nematode strain, Steinernema glaseri SK39 (four technical replicates per strain), with both the $r p o B$ and the $16 \mathrm{~S}$ markers as taxonomic targets. The taxonomic targets were amplified and sequenced and the sequences were processed with the FROGS and DADA2 pipelines. For the rpoB marker, we obtained 48,719 reads (means of 12,180 reads per sample) and 56,699 reads (mean of 14,175 reads per sample) after FROGS and DADA2 processing, respectively (Additional file 6). For $16 \mathrm{~S}$ rRNA gene marker, we obtained 47,298 reads (mean of 11,824 reads per sample) and 41,655 reads (mean of 10,414 reads per sample) after FROGS and DADA2 processing, respectively (Additional file 6).
Rarefaction curves obtained from quality-filtered reads indicated that sequencing depth was sufficient for both the 16S and rpoB markers (Additional file 2). We checked that the biological sample sequences were not contaminated with sequences present in the negative-control samples (Additional file 7). A comparison of the taxonomic affiliation levels of the observed OTUs again confirmed the higher taxonomic discrimination potential of the rрoB marker than of the $16 \mathrm{~S}$ marker (Fig. 1B). For each replicate, OTU numbers after of the application of an abundance threshold of $0.1 \%$ (for individual samples) are detailed in Additional file 6. Depending on the taxonomic marker $(r p o B$ or $16 \mathrm{~S})$ and analysis pipeline (FROGS or DADA2) used, the number of OTUs detected ranged from 30 to 55 (Fig. 6). We then analyzed the 


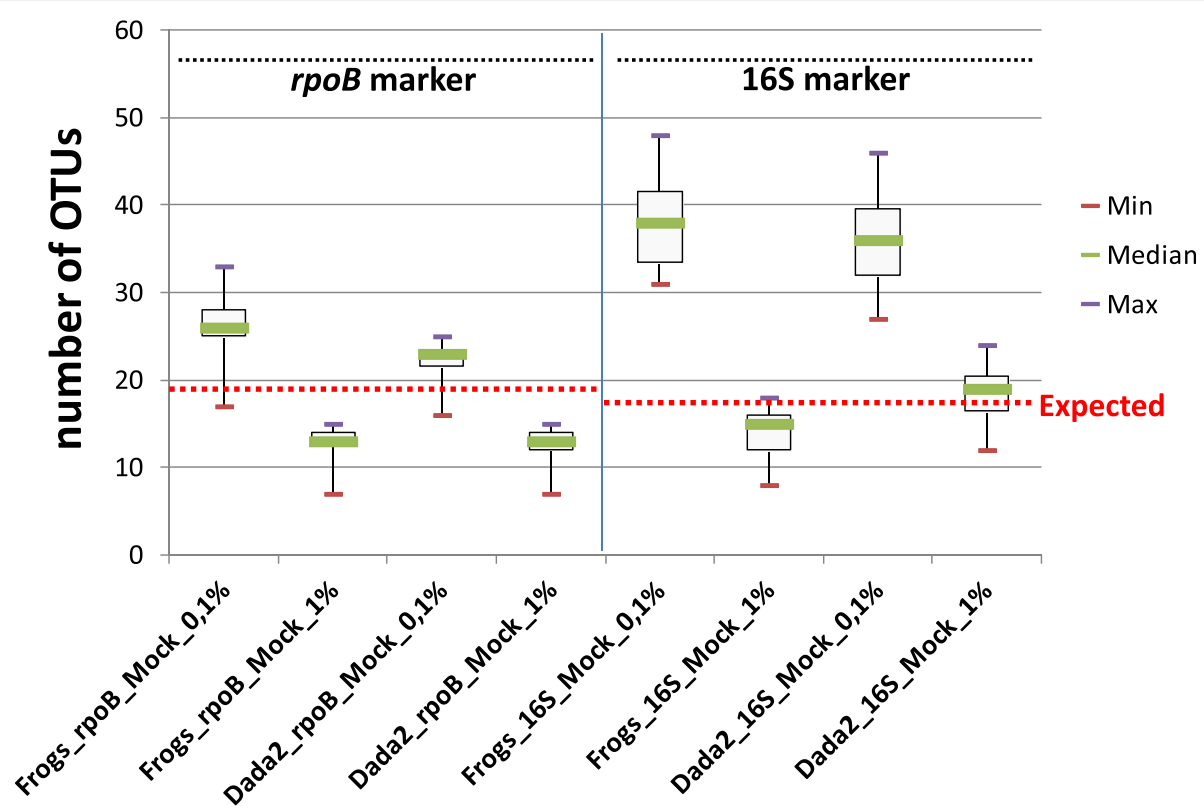

Fig. 2 Impact of the markers, sequence analysis pipeline tools and filtering method on observed OTU richness after Illumina-amplicon sequencing of the five mock communities. Boxplots represent the variation of OTU numbers as a function of: i) Illumina-amplicon sequencing procedure, based on the $r p \circ B$ marker or the 165 marker; ii) sequence analyses process, based on the FROGS or DADA2 pipeline; iii) OTU filtering method based on a $0.1 \%$ read number threshold or a $1 \%$ read number threshold. Each boxplot corresponds to a statistical analysis of the 15 mock community samples (five mock communities, three replicates per mock community); thin purple and red lines correspond to minimum and maximum values, respectively; and the thicker green lines in the boxes correspond to the medians. Red dashed lines correspond to the expected number of OTUs in the mock communities (19 and 17 OTUs for expected_rpoB and expected_16S, respectively)

taxonomic composition of the bacterial communities present in the nematode microbiota. Similar bacterial compositions were obtained with both markers at the phylum level (Fig. 7A and B), with the Proteobacteria the most abundant bacterial phylum. At the family level, the results differed between the two markers (Fig. 7C and D). We refined the comparison of the markers at more discriminant taxonomic ranks, by building phylogenetic trees from OTU sequences (Fig. 8 for FROGS datasets and Additional file 8 for DADA2 dataset). The rpoB marker accurately detected the symbiotic bacterium $X$. poinarii. By contrast, the $16 \mathrm{~S}$ marker predicted the presence of other species from the genera Xenorhabdus and Photorhabdus (phylogenetically close species), leading to the erroneous detection of bacteria that are never associated with this nematode (Fig. 8 and Additional file 8); the symbiotic bacterium $X$. poinarii was identified only by the DADA2 analysis (Additional file 8 ). Moreover, numerous sequence variants were detected with the 16S marker (46 and 40 sequence variants with the FROGS and DADA2 pipelines, respectively), whereas far fewer variants were detected with the $r p o B$ marker (13 and 12 sequence variants with FROGS and DADA2, respectively). We also observed a few OTUs corresponding to chimeras for both markers in DADA2 analysis (Additional file 8). Once these chimeras and sequence variants were removed, the number of OTUs detected in the nematode microbiota was relatively similar between the $16 \mathrm{~S}$ and $r p o B$ markers, at about 25 OTUs (Fig. 8 and Additional file 8 ).

\section{Discussion}

Metabarcoding methods, generally based on the highthroughput sequencing of $16 \mathrm{~S}$ amplicons, are widely used. Despite the known bias of "universal" $16 \mathrm{~S}$ markers, only a few studies have reported the use of alternative markers. We assessed the potential benefit of a portion of the $r p o B$ gene as an alternative genetic marker.

We analyzed the sequence data generated by metabarcoding with rpoB and $16 \mathrm{~S}$ markers on an artificial bacterial DNA complex corresponding to 19 different phylogenetic taxa. One key factor determining the choice of taxonomic markers for metabarcoding studies is the ability of the marker to distinguish between OTUs at the lowest possible taxonomic rank. We found that taxonomic assignation was more accurate with the housekeeping gene $r p o B$ than with the $16 \mathrm{~S}$ marker. The $r p o B$ gene classified OTUs more effectively to species level, with the resolution of the $16 \mathrm{~S}$ marker frequently limited to the genus or a higher taxonomic level. It can be difficult to resolve the taxonomy of 16S rRNA gene sequences based on a limited segment, such as the V3 V4 region. Closely related bacteria, such as those of the 


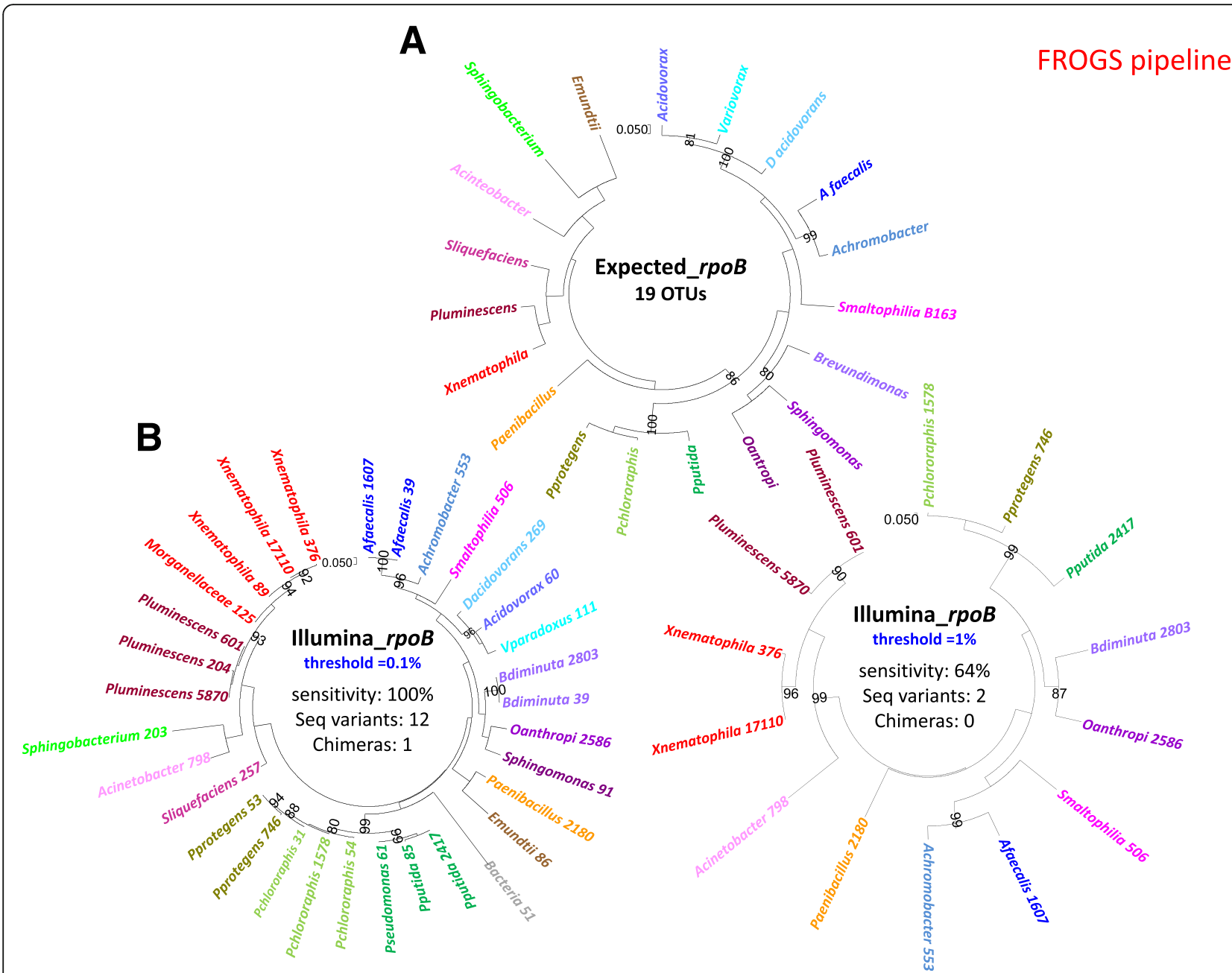

Fig. 3 Comparison of the expected bacterial composition and the observed OTU composition obtained with Illumina-amplicon rpoB sequencing in the mock1 community (FROGS process). Phylogenetic trees based on the rpoB 430 bp-region Muscle alignment were inferred with MEGA7, with a PhyML-based maximum likelihood algorithm and the GTR model for: (a) individual Sanger sequencing of rpoB gene fragment for the 19 taxa making up the experimental mock communities (Expected_rpoB); (b) the observed OTUs obtained after Illumina-amplicon rpoB sequencing of the mock 1 community (OTUs of the three replicates are summed), but only OTUs with an abundance $>0.1 \%$ of total reads in individual replicates were included in the analysis (threshold $=0.1 \%$ ); (c) As in B, but only OTUs with an abundance $>1 \%$ of total reads in individual replicates were included in the analysis (threshold $=1 \%$ ). The OTUs corresponding to the same taxa from the 19 bacterial components of the mock community are highlighted in the same color. The sum of read numbers is indicated after the OTU name. Bootstrap values (percentages of 1000 replicates) of more than $80 \%$ are shown at the nodes

Enterobacteriaceae family, cannot be differentiated solely on the basis of differences in the V3 V4 region [23]. The composition and abundance of OTUs in mock communities are known a priori. Such mock communities are therefore useful tools for detecting potential biases during method development and for optimizing data analysis pipelines [24].

We show here that the rров marker gave results that more closely matched the expected composition of the mock community. When the $16 \mathrm{~S}$ marker was used, we observed a strong distortion of the composition of the bacterial community obtained by the metabarcoding of the mock communities away from the expected bacterial composition, with the values obtained for OTU richness composition higher than the actual OTU richness. This OTU overestimation bias was weaker for the $r p o B$ marker. By contrast to the single-copy housekeeping gene targeted by the rро $B$ marker, sequence heterogeneity between the different copies of the16S marker may lead to the amplification of numerous sequence variants during metabarcoding, resulting in the identification of excessive numbers of OTUs in $16 \mathrm{~S}$ datasets. Intragenomic ribosomal diversity [25] or ribosomal paralogs [26] have frequently been implicated as the major source of sequence variants in $16 \mathrm{~S}$ Illumina 


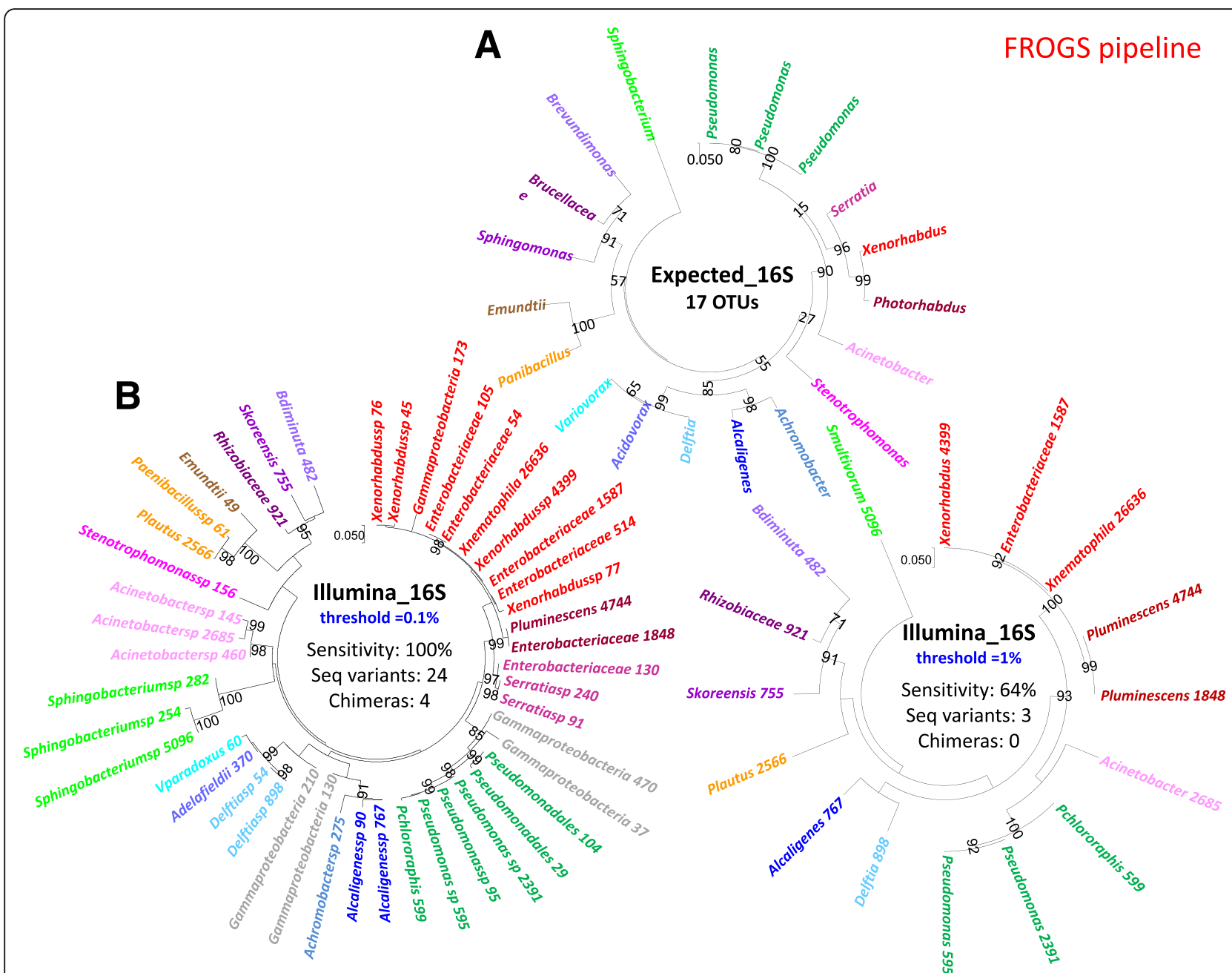

Fig. 4 Comparison of expected bacterial composition and the observed OTU composition generated by Illumina-amplicon $16 \mathrm{~S}$ sequencing for the mock1 community (FROGS process). Phylogenetic trees based on the $16 \mathrm{~S}$ rRNA-V3 V4 region Muscle alignment were inferred with MEGA7, a PhyML-based maximum likelihood algorithm and the GTR model for: (a) individual Sanger sequences of the V3 V4 region of the 165 rRNA gene of the 19 taxa making up the experimental mock communities (Expected_16S); (b) the observed OTUs obtained after Illumina-amplicon 165 sequencing of the mock1 community (OTUs of the three replicates are summed), but only OTUs with an abundance $>0.1 \%$ of total reads in individual replicates were included in the analysis (threshold $=0.1 \%$ ); (c) As in B, but only OTUs with an abundance $>1 \%$ of total reads in individual replicates were included in the analysis (threshold $=1 \%$ ). The OTUs corresponding to the same taxa from the 19 bacterial components of the mock community are highlighted in the same color. The sum of read numbers is indicated after the OTU name. Bootstrap values (percentages of 1000 replicates) of more than $80 \%$ are shown at the nodes

amplicon-sequencing analyses. The observed OTU inflation may also be explained by cumulative errors occurring during the amplification and sequencing steps of the metabarcoding procedure, resulting in the detection of sequence variants. Interestingly, given that $r р о B$ is a protein-coding gene, sequence errors can be readily identified and removed if they disrupt the reading frame [12], providing an added benefit of housekeeping genes as targets in metabarcoding studies. Some authors have also suggested that excessive OTU diversity may be at least partially explained by the presence of unfiltered chimeric reads [27], and laboratory contaminants [28].
Nelson et al. reported a strong overestimation of mock community diversity (25-125 times higher than expected) in the absence of careful checking of the data. Similarly, Kunin et al. [29] found that diversity was grossly overestimated for their mock community data unless a quality threshold was implemented. We show here that the removal of OTUs with read abundances below 1\% (for individual samples) decreases the number of sequence variants. However, this threshold cutoff of $1 \%$ is much less sensitive than the $0.1 \%$ threshold cutoff for describing the bacterial communities making up the mock communities. 

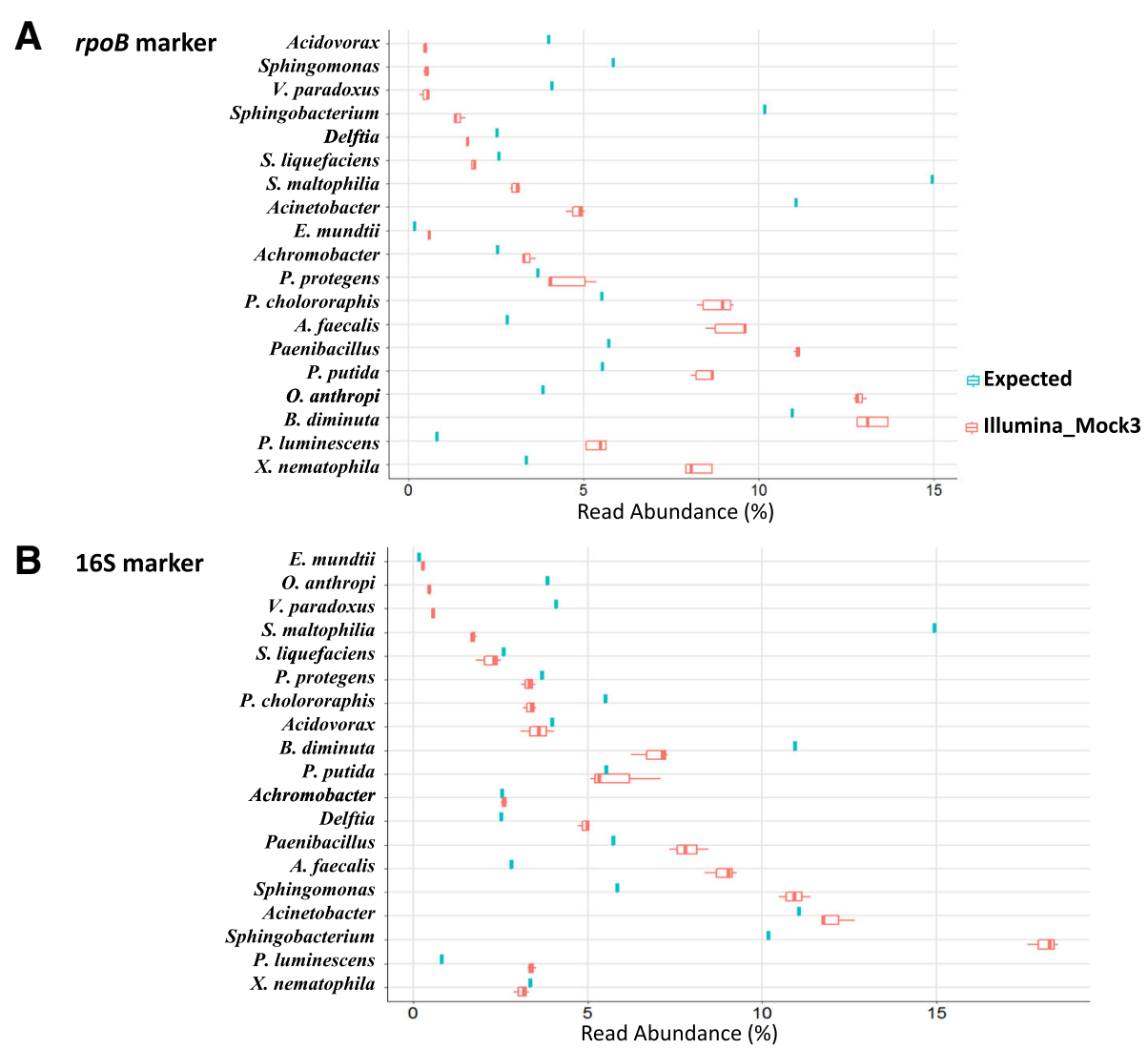

Fig. 5 Comparison of the observed and expected relative abundances of the bacterial community of the mock3. Boxplots show the variation of OTU relative abundance in: (a) the mock3 community (three replicates), with use of the rpoB marker; (b) the mock3 community (three replicates) with use of the 165 marker. The blue box plots show the expected relative abundances of the species based on the theoretical composition of the mock3 community, and the red box plots show the observed relative abundance of OTUs based on Illumina-amplicon sequencing of the mock3 community (FROGS pipeline analysis). Error bars indicate the standard deviation for triplicate samples. The taxonomic identities of the 19 bacterial strains making up the mock 3 community are indicated on the $y$-axis, and the relative abundance of each taxon (values = median percentage read abundance across all replicates for Illumina-sequenced mock communities) is plotted on the $x$-axis. The number of reads has been corrected with respect to the number of copies of the 165 rRNA gene in each taxon, for the calculation of observed relative abundance

When using metabarcoding for quantitative analyses, caution is required concerning the conclusions drawn about the relative abundances of bacterial taxa, even with a single-copy gene, such as rpoB. Our results highlight the existence of a bias in abundance taxa, this bias being strain-dependent and varying with the marker used. This bias probably reflects the amplification bias occurring during PCR cycles [6].

We finally used the rpoB marker to analyze microbial communities in an entomopathogenic nematode, Steinernema glaseri, known to carry an intestinal symbiotic bacterium, Xenorhabdus poinarii, but for which microbiota composition remains otherwise unknown. We found that the findings concerning the bacterial community associated with $S$. glaseri depended on the marker used. The rpoB marker gave better taxonomic discrimination and was capable of reliably identifying the symbiotic bacterium. The $16 \mathrm{~S}$ rRNA marker is able to detect the symbiont only with one of the pipelines, as well as false positive OTUs phylogenetically related to the symbiont.

The $r р о B$ marker was successfully used here to describe the microbiota of an entomopathogenic nematode, with a microbiota of low diversity including bacterial taxa for which $r p o B$ sequences are routinely available from databases. Further testing of the $r p o B$ marker is required in other types of complex microbiota, such as those containing members of phyla other than Firmicutes and Proteobacteria. Despite its disadvantages, the $16 \mathrm{~S}$ marker should not be entirely abandoned, particularly for the exploration of complex and unknown ecological niches, as it is a 
Table 3 Abundance bias ratios calculated for the 19 taxa after Illumina-amplicon sequencing of rpoB and 16S rRNA for the five mock communities

\begin{tabular}{|c|c|c|c|c|c|c|c|c|c|c|}
\hline & \multirow[b]{3}{*}{$16 S$ copy number } & \multirow[b]{3}{*}{ Genome size (Mb) } & \multicolumn{8}{|c|}{ Ratio of metabarcoding relative abundance of OTUs / expected relative abundance } \\
\hline & & & \multicolumn{2}{|l|}{ Mock1 } & \multicolumn{2}{|c|}{ Mock3 } & \multicolumn{2}{|c|}{ Mock4 } & \multicolumn{2}{|l|}{ Mock5 } \\
\hline & & & rpoB & $16 S$ & rрoB & $16 \mathrm{~S}$ & rpoB & $16 \mathrm{~S}$ & rров & $16 \mathrm{~S}$ \\
\hline E. mundtii & 6 & 3.3 & 2.21 & 0.91 & 3.44 & 1.45 & 3.61 & 1.15 & 4.21 & 1.61 \\
\hline D. acidovorans & 5 & 6.8 & 0.46 & 1.42 & 0.66 & 1.86 & 0.75 & 2.03 & 0.82 & 1.93 \\
\hline A. xyloxisidans & 3 & 6.8 & 0.93 & 0.68 & 1.32 & 0.97 & 1.35 & 0.94 & 1.42 & 0.85 \\
\hline S. liquefaciens & 7 & 5.3 & 0.43 & 0.34 & 0.71 & 0.82 & 0.79 & 0.91 & 0.87 & 0.97 \\
\hline A. faecalis & 3 & 4.2 & 2.52 & 2.37 & 3.29 & 3.15 & 3.58 & 3.34 & 3.85 & 3.31 \\
\hline P. protegens & 5 & 7.0 & 0.94 & 0.60 & 1.27 & 0.85 & 1.25 & 0.87 & 1.30 & 0.88 \\
\hline O. anthropi & 4 & 5.2 & 2.89 & 1.22 & 3.33 & 1.59 & 3.67 & 1.64 & 3.64 & 1.68 \\
\hline Acidovorax sp & 3 & 4.9 & 0.06 & 0.58 & 0.12 & 0.86 & 0.14 & 1.00 & 0.16 & 0.98 \\
\hline V. paradoxus & 2 & 7.5 & 0.12 & 0.14 & 0.11 & 0.13 & 0.14 & 0.17 & 0.07 & 0.12 \\
\hline P. chlororaphis & 5 & 7.3 & 1.29 & 0.40 & 1.61 & 0.57 & 1.72 & 0.56 & 1.74 & 0.60 \\
\hline P. putida & 7 & 6.2 & 1.97 & 1.42 & 1.52 & 0.92 & 1.62 & 1.14 & 1.71 & 1.07 \\
\hline Paenibacillus sp & 10 & 6.0 & 1.68 & 0.84 & 1.99 & 1.25 & 2.23 & 1.17 & 2.21 & 1.28 \\
\hline Sphingomonas sp & 2 & 4.1 & 0.07 & 1.26 & 0.09 & 1.75 & 0.09 & 1.90 & 0.08 & 1.78 \\
\hline Sphingobacterium sp & 7 & 6.9 & 0.09 & 1.40 & 0.14 & 1.68 & 0.19 & 1.62 & 0.18 & 1.65 \\
\hline Brevundimonas sp. & 2 & 3.4 & 1.13 & 0.41 & 1.21 & 0.60 & 1.30 & 0.59 & 1.25 & 0.57 \\
\hline Acinetobacter sp. & 6 & 3.6 & 0.31 & 0.76 & 0.43 & 1.02 & 0.53 & 0.98 & 0.56 & 1.03 \\
\hline S. maltophilia & 4 & 4.9 & 0.14 & 0.06 & 0.20 & 0.11 & 0.23 & 0.09 & 0.19 & 0.09 \\
\hline P. luminescens & 7 & 5.7 & 1.56 & 0.97 & 6.66 & 4.01 & 5.60 & 2.97 & 10.76 & 3.63 \\
\hline X. nematophila & 7 & 4.6 & 1.18 & 1.37 & 2.42 & 0.95 & 2.57 & 0.76 & 2.68 & 1.16 \\
\hline
\end{tabular}

reference marker for which a very rich and complete database is available for taxonomic assignment. For highthroughput amplicon sequencing studies, we therefore recommend the use of multigenic approaches based on different taxonomic markers, targeting at least one housekeeping gene (rpoB is a good candidate) and a variable $16 \mathrm{~S}$ rRNA gene region.

\section{Conclusions}

The use of $16 \mathrm{~S}$ sequencing raises a number of challenges, including primer bias, gene copy number, PCR or sequencing artifacts and contamination. Metabarcoding approaches, which target a taxonomically relevant marker, such as the $r p o B$ gene, are a potential alternative, making it possible to overcome at least some of these challenges. The major benefit of rpoB sequencing is its potential for improving taxonomic assignment and for more detailed investigations of OTU richness at species level, providing a more accurate description of the composition of microbiota communities. The greater ability of rpoB-based analyses to discriminate between phylogenetically different groups of species should increase resolution and provide more reliable results for metagenomic studies. Our results also highlight the need to develop and use mock community as controls for all microbial studies, to pick up potential sequence errors, which may arise at any step in next-generation sequencing protocols. For Illumina amplicon-sequencing strategies, we also strongly recommended the use of a multigenic approach based on at least two taxonomic markers, including a protein-coding gene, such as $r p o B$, and the $16 \mathrm{~S}$ rRNA marker.

\section{Methods \\ Biological material}

The nematode/bacterial strains and primers used in this study are listed in Additional file 9.

Isolation, multiplication and storage of nematode strains Steinernema nematodes were originally isolated with an ex situ Galleria trap, as previously described [30]. The nematodes used here had been stored for decades in the DGIMI collection. Infective juveniles (IJs) were stored in Ringer's solution (Merck) and were multiplied every six months by infestation of the last instar of Galleria. Briefly, IJs were added to Galleria larvae in Petri dishes (laboratory Galleria trap). When the Galleria larvae died, their cadavers were placed on a white trap and the IJs that 


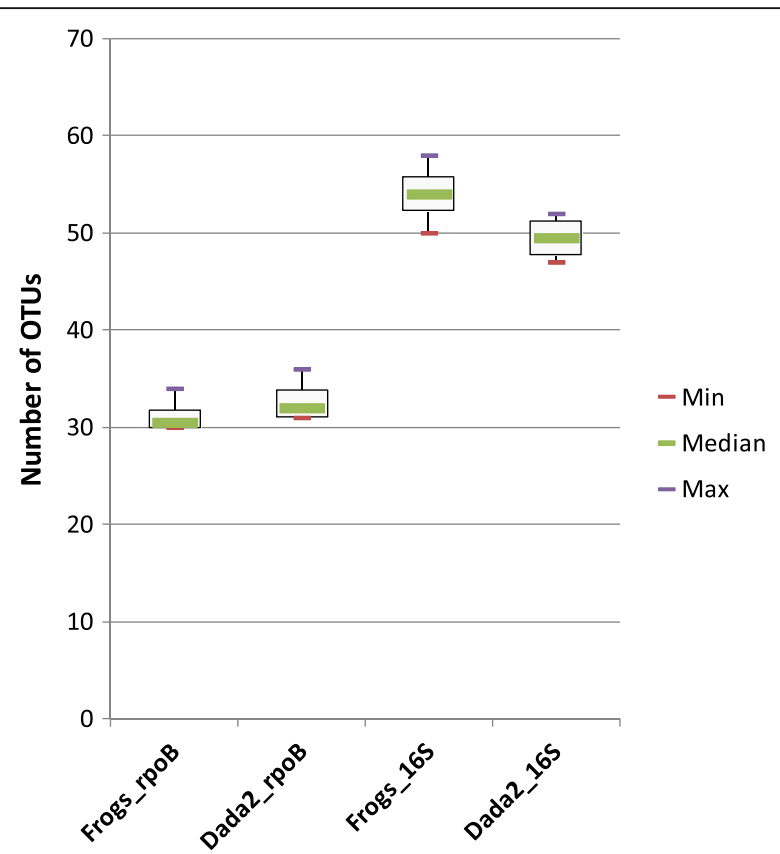

Fig. 6 Impact of the markers and sequence analysis pipeline tool used on observed OTU richness after Illumina-amplicon sequencing of the nematode Steinernema glaseri. Boxplots representing the variation in OTU numbers (only OTUs with an abundance $>0.1 \%$ of total reads in individual replicates) as a function of the marker (rpoB marker versus 165 marker) and sequence analyse pipeline tool (FROGS versus DADA2) used. Each boxplot corresponds to a statistical analysis of the four replicates; thin purple and red lines correspond to minimum and maximum values, respectively; and thicker green lines in the boxes correspond to the medians

emerged from them were stored in Ringer's solution at $9{ }^{\circ} \mathrm{C}$.

Isolation, multiplication and storage of the bacteria used in the mock communities

The symbiotic bacteria, Xenorhabdus and Photorhabdus, were isolated by the hanging drop technique [31]. Bacteria from other genera were isolated from crushed IJs nematodes or from the contents of G. mellonella cadavers after nematode infestation. For the isolation of bacteria from entomopathogenic nematodes, 20 IJs were placed in a $1.5 \mathrm{~mL}$ Eppendorf tube containing $200 \mu \mathrm{L}$ of Lysogeny Broth medium and three 3-mm glass beads, and were subjected to three cycles of grinding $(1 \mathrm{~min}$, at $30 \mathrm{~Hz}$ followed by 1 min without agitation) in a TissueLyser II apparatus (Qiagen, France). The solutions obtained from crushed nematodes or the contents of G. melonella were plated on both nutrient agar (Difco) plates and nutrient bromothymol blue agar (NBTA) plates [32], and incubated at $28^{\circ} \mathrm{C}$ for 48 h. Bacterial colonies with different morphotypes were stored at $-80^{\circ} \mathrm{C}$ in $16 \%$ glycerol (v/v).
Molecular identification of the bacteria isolated and mock community design

Bacterial isolates were identified as previously described [33], by amplifying and sequencing a near full-length $16 \mathrm{~S}$ rRNA gene (1372 bases). Briefly, bacterial genomic DNA was extracted as previously described [34] and stored at $4{ }^{\circ} \mathrm{C}$. The $16 \mathrm{~S}$ rRNA gene was amplified (the primer sequences are indicated in Additional file 9) in a Bio-Rad thermocycler (BioRad, USA). PCR products were analyzed by agarose gel electrophoresis. Sanger sequencing of the 16S rRNA amplicons was performed by MWG-Eurofins (Deutschland), and sequences were blasted against the NCBI database for taxonomic identification of the bacterial isolates. Nineteen bacterial isolates encompassing a broad taxonomic diversity among eubacteria were selected to compose the reference mock communities (Table 1). The DNA concentration of each selected bacterial strain was quantified on a Qubit Fluorometer (Thermo Fisher Scientific, USA) and five mock communities were generated by mixing the DNA of the 19 strains (the DNA concentrations of symbiotic taxa in the mock communities varied over four orders of magnitude). The proportions of the 19 bacterial taxa composing the five mock communities, in genome number equivalents, are shown in Table 2. The number of genome equivalents was calculated as follows: number of genome copies $=[\mathrm{DNA}$ amount $(\mathrm{ng}) * 6.022 \times 10^{23}$ ] / [Genome size (bp) * $1 \times 10^{9} *$ $650 \mathrm{~g} / \mathrm{mole}$ of bp].

Sanger sequencing of the $r p o B$ and V3 V4 regions of the 19 bacterial isolates present in the mock communities

The $r p o B$ and V3 V4 regions were amplified as described above (the primer sequences are indicated in Additional file 9). Sanger sequencing of the amplicons was performed by MWG-Eurofins (Deutschland).

\section{DNA extraction from IJs}

We performed four DNA extraction replicates per nematode sample, each replicate consisting of about five thousand IJs sampled from a storage batch. We minimized the risk of contamination with microorganisms from the body surface, by washing the IJs with copious amounts of tap water on a filter. The washed IJs were recovered from the filter with a sterile pipette, transferred to $10 \mathrm{~mL}$ of sterile ultrapure water and immediately frozen at $-80^{\circ} \mathrm{C}$ for future use. DNA was extracted from the IJs with the Quick Extract kit (Epi-centre, USA). Briefly, frozen samples were rapidly thawed, heated at $80{ }^{\circ} \mathrm{C}$ for $20 \mathrm{~min}$ and centrifuged $(2,500 \mathrm{x} \mathrm{g}, 10 \mathrm{~min})$ to pellet the IJs. Once the supernatant had been removed, we added $200 \mu \mathrm{L}$ of Quick Extract lysis solution and the mixture was transferred to $2 \mathrm{~mL}$ Eppendorf tubes containing three sterile 3-mm glass beads. IJs were crushed by three cycles of mechanical grinding $(2 \mathrm{~min}$ at $30 \mathrm{~Hz}$ 


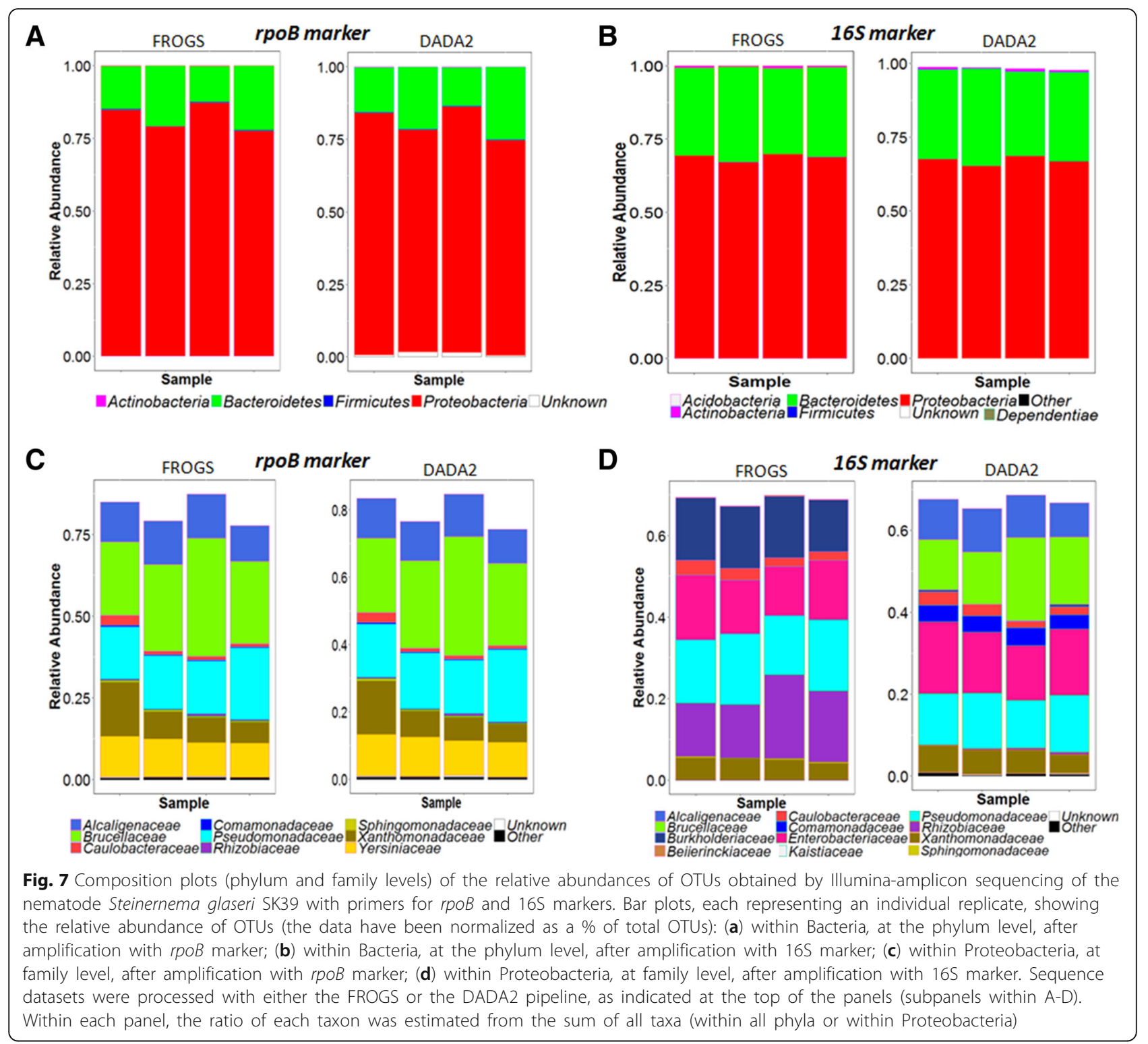

followed by $1 \mathrm{~min}$ without agitation) in a TissueLyser II apparatus (Qiagen, France). We added $2 \mu \mathrm{L}$ of ReadyLyse Lysozyme Solution (Epi-centre, USA) to the ground samples, which were then incubated at room temperature until the solution cleared (24 to $48 \mathrm{~h}$ ). Samples were again heated at $80^{\circ} \mathrm{C}$ for $20 \mathrm{~min}$, and the lysis of IJs was checked under a light microscope. After complete lysis, the samples were subjected to an additional treatment with $20 \mu \mathrm{L}$ of $20 \mathrm{mg} / \mathrm{mL}$ RNaseA (Invitrogen PureLinkTM RNaseA, France). Finally, a phenol-chloroform purification step was performed, followed by chloroform purification. The DNA was precipitated in absolute ethanol, washed twice in 70\% ethanol, resuspended in $50 \mu \mathrm{L}$ ultrapure water and stored at $-20^{\circ} \mathrm{C}$. The DNA products were analyzed by agarose gel electrophoresis and quantified with a Nanodrop spectrophotometer (Thermo Fisher Scientific). We assessed the levels of contaminating bacterial DNA at each of the various steps in DNA sample preparation, by including several negative control extractions with sterile ultra-pure water.

\section{Design of $r p o B$ primers suitable for use in MiSeq Illumina sequencing}

Degenerate consensual pairs of rров primers called Univ_rpoB_F_deg (forward primer) and Univ_rpoB_ R_deg (reverse primer) were manually designed from clustalW alignments (http://multalin.toulouse.inra.fr/ 

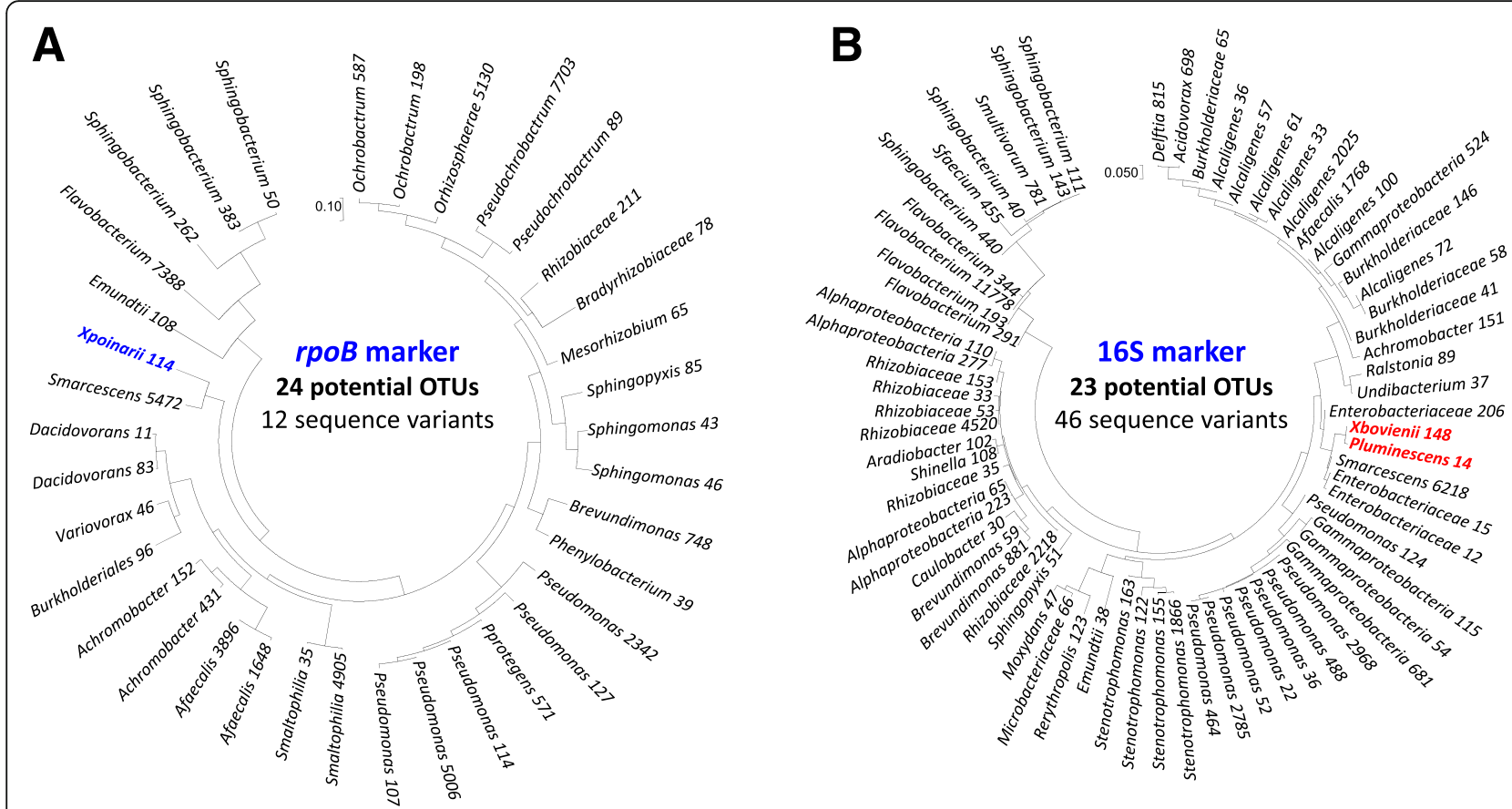

Fig. 8 Comparison of the bacterial composition data generated by Illumina sequencing of the nematode Steinernema glaseri SK39 (FROGS process) with the rpoB and 165 markers. Phylogenetic trees were inferred with MEGA7, using a PhyML-based maximum likelihood algorithm and the GTR model: (a) Phylogenetic tree of the observed OTUs obtained after Illumina-amplicon rpoB sequencing; (b) Phylogenetic tree of the observed OTUs obtained after Illumina-amplicon 165 rRNA sequencing. The OTUs of the three replicates are summed, but only OTUs with an abundance $>0.1 \%$ of total reads in individual replicates were included in the analysis. The OTUs corresponding to the true symbiotic bacterium (X. poinarii) are highlighted in blue. The OTUs corresponding to false-positive symbiotic bacteria from the genera Xenorhabdus and Photorhabdus are highlighted in red. The sum of the read numbers is indicated after the OTU name. Bootstrap values (percentages of 1000 replicates) of more than $80 \%$ are shown at the nodes

multalin/) of rpoB gene sequences from bacterial reference genomes covering a broad taxonomic diversity among eubacteria. The binding sites of the selected primers correspond to Escherichia coli K12 nucleotide positions 1630 to 2063, making it possible to amplify a 434-nucleotide portion of the rpoB gene. The specificity of the $r p o B$ primers was checked in silico on a large panel of publicly available complete sequenced genomes with the "Blast and Pattern Search" web tool implemented on the MaGe annotation platform (http://www.genoscope. cns.fr/agc/mage). The universality of the primers was checked in silico on a large panel of $r p o B$ genomic sequences publicly available from the NCBI database, with the Primers toolbox implemented in CLC Genomics Workbench 3.6.1.

\section{Library preparation and Illumina MiSeq sequencing}

Amplicon libraries were constructed following two rounds of PCR amplification. The first amplification step was performed with the high-fidelity iProof $^{\text {mix }}$ DNA Polymerase (BioRad), in a Bio-Rad thermocycler, on 10 to $100 \mathrm{ng}$ of DNA. The hypervariable V3 V4 region of the $16 \mathrm{~S}$ rRNA gene was targeted with the universal primers $\mathrm{F} 343$ and R784, and the rpoB fragment was targeted with the previously designed primers Univ_rpoB_F_deg and Univ_rpoB_R_deg (the primer sequences are shown in Additional file 9). Thirty amplification cycles were performed with annealing temperatures of $65{ }^{\circ} \mathrm{C}$ and $60^{\circ} \mathrm{C}$ for the $\mathrm{V} 3 \mathrm{~V} 4$ region ( 460 base pairs), and the rpoB region $(\sim 435$ base pairs), respectively. We assessed the amount of contaminating DNA in these PCRs, by including negative PCR controls with sterile ultra-pure water as the template. The amounts of amplicon DNA and amplicon sizes were analyzed by agarose gel electrophoresis. Single multiplexing was performed at the Genomics and Transcriptomics Platform (INRA, Toulouse, France), with 6 base pairs index sequences, which were added to reverse primers during a second PCR with 12 cycles. Amplicon libraries were sequenced with Illumina MiSeq technology (MiSeq Reagent Kit v2) according to the manufacturer's instructions. FastQ files were generated at the end of the run. The FastQC program [35] was used for quality control on raw sequence data and the quality of the run was checked internally, by adding 30\% PhiX sequences at a 
concentration of $12.5 \mathrm{pM}$. Each paired-end sequence was assigned to its sample with the previously integrated index, and paired-end reads were assembled with FLASH [36]. Unassembled reads were discarded. The raw sequence data can be downloaded from http://www.ebi.ac.uk/ena/ (accession numbers: PRJEB24936 for the mock communities; ERS2715153ERS2715156 and ERS2715252-ERS2715255 for the nematode samples; ERS2715166-ERS2715174 and ERS2715260ERS2715268 for the extraction-control samples).

\section{rpoB sequence database design}

For taxonomic assignment with the rpoB marker, we constructed a reference database including $\sim 45000$ sequences; this database is available from the FROGS website (http://frogs.toulouse.inra.fr/).

The $г$ rо $B$ sequences were collected and the quality of the resulting database was checked as previously described [9] for the $g y r B$ database. Briefly, $r p o B$ sequences were retrieved from 47,175 genomes sequences publicly available from the IMG database [37] at the time of analysis. CDSs belonging exclusively to the TIGR02013 protein family were defined as $\mathrm{RpoB}$ homologs and were retrieved for further analysis (approximately 46,300 hits found in 45, 500 genomic sequences). The corresponding nucleotide sequences of the selected region used for Illumina sequencing (434 nucleotides - see above) were aligned as previously described [9]; redundant and aberrant sequences (sequences containing ambiguous nucleotides or with sequences of less than 430 nucleotides) were removed from the database.

\section{Bioinformatic processing of sequence data}

The sequence reads obtained were processed according to the FROGS (Find Rapidly OTUs with Galaxy Solution) pipeline [38] and the DADA2 pipeline [39].

\section{The FROGS process}

A preprocessing tool was used to remove sequences not bound to both primers, to trim the primers, and to remove all sequences containing an ambiguous base. Sequence clustering was performed with the Swarm algorithm [40]. Chimera sequences were detected with the VSEARCH algorithm, by the de novo UCHIME method [41, 42], and were removed. A filtering tool was used to remove spurious clusters, with read abundances of less than $0.005 \%$ of the total number of reads. The filtered sequences were assigned to taxa with RDP Classifier [43] and the 16S rRNA database SILVA pintail quality $100 \%$ [3] for V3 V4 reads and the rpoB database for $r p o B$ reads. The sequences were clustered into OTUs with a $97 \%$ similarity cutoff (with a bootstrap confidence of $80 \%$ ). The OTU abundance tables are available in Additional file 10.

\section{The DADA2 process}

The DADA2 method was developed for the analysis of short-read amplicon sequences [39]. The pipeline is based on a complete bioinformatic workflow including quality filtering, dereplication, sample inference, chimera removal, and optionally, a taxonomic assignment step. The DADA2 software takes raw amplicon sequencing data in fastq files as input, and produces an error-corrected table of the abundances of amplicon sequence variants in each sample (an ASV table) as output. As for the FROGS process, the sequence variants were assigned to taxa with RDP Classifier (sequence similarity threshold $=97 \%$, bootstrap confidence cutoff $=80 \%$ ) and the $16 \mathrm{~S}$ rDNA database SILVA [3] for V3 V4 reads and the $r p o B$ database for $r p o B$ reads. The OTU abundance tables are available in Additional file 10.

\section{Bacterial community and statistical analyses}

OTU diversity and statistical analyses were performed with the R packages Phyloseq [44], Vegan [45], and Ampvis 2 (https://madsalbertsen.github.io/ampvis2/). Briefly, rarefaction curves were calculated with Phyloseq $\mathrm{R}$ packages. Beta diversity was analysed with custom-developed Phyloseq command lines. A PCoA analysis (Ampvis $2 \mathrm{R}$ package) based on the Bray-Curtis dissimilarity matrix was used to visualize the differences between the microbial communities of the nematodes and the microbial contaminants associated with the extraction of control samples. The significance of the clustering on PCoA plots was assessed by multivariate PERMANOVA in the Phyloseq $R$ package on a Bray-Curtis similarity matrix with a type III of sum of squares, 9999 permutations and unrestricted permutations of raw data. The amp_boxplot function (Ampvis2 R package) was used to generate boxplots of the OTU relative abundances. All the $\mathrm{R}$ plots in the study were generated with the ggplot2 R package [46].

\section{Phylogenetic analysis}

Phylogenetic analysis of the V3 V4 and rpoB amplicons, sequence alignment and maximum likelihood analysis with the Generalised time-reversible (GTR) model were performed as described elsewhere [22]. The Mega7 tool [47] was used to generate circular phylogenetic trees.

\section{Additional files}

Additional file 1: Sequence reads and OTU numbers obtained by Illumina-amplicon sequencing for rpoB and 165 markers in 15 mock community samples (five mock communities, three replicates per mock community). (DOCX $27 \mathrm{~kb}$ )

Additional file 2: Rarefaction curves obtained by Illumina-amplicon sequencing of rpoB (A) and $16 \mathrm{~S}(\mathrm{~B})$ markers in 15 mock community samples (five mock communities, three replicates per mock) and the four replicates of the Steinernema glaseri nematode sample. (PPTX $100 \mathrm{~kb}$ ) 
Additional file 3: Comparison of the expected bacterial composition and the observed OTU composition generated by Illumina-amplicon rpoB sequencing for the mock1 community (DADA2 process). See Fig. 3 for Figure legend details. (PPTX $250 \mathrm{~kb}$ )

Additional file 4: Comparison of expected bacterial composition and the observed OTU composition obtained by Illumina-amplicon 165 rRNA gene sequencing for the mock1 community (DADA2 process). See Fig. 4 for Figure legend details. (PPTX 296 kb)

Additional file 5: Comparison of the observed and expected relative abundances of the bacterial communities obtained by Illumina-amplicon $\operatorname{rpoB}(\mathrm{A})$ and $16 \mathrm{~S}(\mathrm{~B})$ sequencing for the mock1, mock2, and mock3 communities. See Fig. 5 for Figure legend details. (PPTX 288 kb)

Additional file 6: Sequence reads and OTU numbers obtained by Illumina-amplicon sequencing of rpoB and 165 markers for the nematode samples (four replicates). (DOCX $24 \mathrm{~kb}$ )

Additional file 7: Comparison of the bacterial communities associated with nematode samples (Steinernema glaseri SK39) and extraction control samples. (PPTX $80 \mathrm{~kb}$ )

Additional file 8: Comparison of the bacterial compositions obtained by Illumina sequencing of $r p o B$ and 165 rRNA for the nematode S. glaseri SK39 (DADA2 process). See Fig. 8 for Figure legend details. (PPTX $245 \mathrm{~kb}$ )

Additional file 9: List of biological materials used in the experimental study (DOCX $39 \mathrm{~kb})$

Additional file 10: OTU abundance tables after Illumina-amplicon sequencing of $r$ POB or 165 markers and use of the FROGS or DADA2 sequence analysis pipeline. (XLSX $1120 \mathrm{~kb}$ )

\section{Abbreviations}

BLAST: Basic Local Alignment Search Tool; DNA: Deoxyribonucleic acid; EMBL: European Molecular Biology Laboratory; FROGS: Find Rapidly OTUs with Galaxy Solution; GTR model: Generalised time-reversible model; IJs: Infective Juveniles; NBTA: Nutrient bromothymol blue agar; NCBI: National Center for Biotechnology Information; OTU: Operational taxonomic unit; PCR: Polymerase chain reaction; RDP Classifier: Ribosomal Database Project (RDP) Classifier

\section{Acknowledgments}

The authors thank Alain Givaudan and Julien Brillard for the proofreading of the manuscript.

\section{Authors' contributions}

JCO and SG conceived the study. JCO and SP designed and performed the experiments. JCO, SP, MG, MB and SG analyzed the data. JCO and SG supervised the project. All authors wrote, read and approved the manuscript.

\section{Funding}

This work was funded by Health Plant and Environment Department of INRA (grant 2015-2017) and the MEM-INRA metaprogram (grant P10016).

\section{Availability of data and materials}

The datasets generated and analyzed in this study are available from the ENA (European Nucleotide Archive) repository (http://www.ebi.ac.uk/ena/ data/view/PRJEB24936).

\section{Ethics approval and consent to participate}

Not applicable.

\section{Consent for publication}

Not applicable.

\section{Competing interests}

The authors have no competing interests to declare.

\section{Author details}

'DGIMI, INRA, University Montpellier, 34095 Montpellier, France. ${ }^{2}$ INRA AGROCAMPUS-Ouest, Universite d'Angers, Beaucouze, France. ${ }^{3}$ CBGP INRA CIRAD IRD Montpellier SupAgro, Univ. Montpellier, 755 avenue du Campus Agropolis, CS 300 16, F-34988 Montferrier sur Lez cedex, France.
Received: 27 March 2019 Accepted: 18 July 2019

Published online: 29 July 2019

\section{References}

1. DeSantis TZ, Hugenholtz P, Larsen N, Rojas M, Brodie EL, Keller K, et al. Greengenes, a chimera-checked 16S rRNA gene database and workbench compatible with ARB. Appl Environ Microbiol. 2006:72:5069-72.

2. Cole JR, Tiedje JM. History and impact of RDP a legacy from Carl Woese to microbiology. RNA Biol. 2014;11:239-43.

3. Quast C, Pruesse E, Yilmaz P, Gerken J, Schweer T, Yarza P, et al. The SILVA ribosomal RNA gene database project: improved data processing and web-based tools. Nucleic Acids Res. 2013;41:D590-6.

4. Schirmer M, ljaz UZ, D'Amore R, Hall N, Sloan WT, Quince C. Insight into biases and sequencing errors for amplicon sequencing with the Illumina MiSeq platform. Nucleic Acids Res. 2015;43:e37.

5. Kennedy K, Hall MW, Lynch MD, Moreno-Hagelsieb G, Neufeld JD. Evaluating bias of Illumina-based bacterial 165 rRNA gene profiles. Appl Environ Microbiol. 2014:80:5717-22.

6. Schloss PD, Gevers D, Westcott SL. Reducing the effects of PCR amplification and sequencing artifacts on 165 rRNA-based studies. PLoS One. 2011;6:e27310.

7. Roux S, Enault F, Bronner G, Debroas D. Comparison of $16 \mathrm{~S}$ rRNA and protein-coding genes as molecular markers for assessing microbial diversity (Bacteria and archaea) in ecosystems. FEMS Microbiol Ecol. 2011;78:617-28.

8. Poirier S, Rue O, Peguilhan R, Coeuret G, Zagorec M, Champomier-Verges MC, et al. Deciphering intra-species bacterial diversity of meat and seafood spoilage microbiota using gyrB amplicon sequencing. A comparative analysis with 16S rDNA V3-V4 amplicon sequencing. PLoS One. 2018;13:e204629.

9. Barret M, Briand M, Bonneau S, Preveaux A, Valiere S, Bouchez O, et al. Emergence shapes the structure of the seed microbiota. Appl Environ Microbiol. 2015;81:1257-66.

10. Moeller AH, Caro-Quintero A, Mjungu D, Georgiev AV, Lonsdorf EV, Muller MN, et al. Cospeciation of gut microbiota with hominids. Science. 2016;353:380-2

11. Case RJ, Boucher Y, Dahllof I, Holmstrom C, Doolittle WF, Kjelleberg S. Use of $16 \mathrm{~S}$ rRNA and rpoB genes as molecular markers for microbial ecology studies. Appl Environ Microbiol. 2007;73:278-88.

12. Vos M, Quince C, Pijl AS, de Hollander M, Kowalchuk GA. A comparison of $r p o B$ and 165 rRNA as markers in pyrosequencing studies of bacterial diversity. PLoS One. 2012:7:e30600.

13. Ghebremedhin B, Layer F, Konig W, Konig B. Genetic classification and distinguishing of Staphylococcus species based on different partial gap, $16 \mathrm{~S}$ rRNA, hsp60, rpoB, sodA, and tuf gene sequences. J Clin Microbiol. 2008;46:1019-25.

14. Links MG, Dumonceaux TJ, Hemmingsen SM, Hill JE. The chaperonin-60 universal target is a barcode for bacteria that enables de novo assembly of metagenomic sequence data. PLoS One. 2012;7:e49755.

15. Adekambi T, Drancourt M, Raoult D. The rpoB gene as a tool for clinical microbiologists. Trends Microbiol. 2009;17:37-45.

16. Adekambi T, Shinnick TM, Raoult D, Drancourt M. Complete rpoB gene sequencing as a suitable supplement to DNA-DNA hybridization for bacterial species and genus delineation. Int J Syst Evol Microbiol. 2008:58:1807-14

17. Drancourt M, Raoult D. rpoB gene sequence-based identification of Staphylococcus species. J Clin Microbiol. 2002:40:1333-8.

18. Mollet C, Drancourt M, Raoult D. rpoB sequence analysis as a novel basis for bacterial identification. Mol Microbiol. 1997:26:1005-11.

19. Brivio MF, Mastore M. Nematobacterial complexes and insect hosts: different weapons for the same war. Insects. 2018;9(3):117-30.

20. Nielsen-LeRoux C, Gaudriault S, Ramarao N, Lereclus D, Givaudan A. How the insect pathogen bacteria Bacillus thuringiensis and Xenorhabdus/ Photorhabdus occupy their hosts. Curr Opin Microbiol. 2012;15:220-31.

21. Akhurst RJ. Xenorhabdus nematophilus subsp poinarii: its interaction with insect pathogenic nematodes. Syst Appl Microbiol. 1986;8:142-7.

22. Ogier JC, Pages S, Bisch G, Chiapello H, Medigue C, Rouy Z, et al. Attenuated virulence and genomic reductive evolution in the entomopathogenic bacterial symbiont species, Xenorhabdus poinarii. Genome Biol Evol. 2014;6:1495-513.

23. Jovel J, Patterson J, Wang W, Hotte N, O'Keefe S, Mitchel T, et al. Characterization of the gut microbiome using $16 \mathrm{~S}$ or shotgun metagenomics. Front Microbiol. 2016;7:459. 
24. Yeh YC, Needham DM, Sieradzki ET, Fuhrman JA. Taxon Disappearance from Microbiome Analysis Reinforces the Value of Mock Communities as a Standard in Every Sequencing Run. 2018;3(3):e00023-18.

25. Krohn A, Stevens B, Robbins-Pianka A, Belus M, Alla GJ, Gehring C. Optimization of 165 amplicon analysis using mock communities: implications for estimating community diversity. PeerJ Preprints. 2016;4:e2196v3.

26. Pei AY, Oberdorf WE, Nossa CW, Agarwal A, Chokshi P, Gerz EA, et al. Diversity of 16S rRNA genes within individual prokaryotic genomes. Appl Environ Microbiol. 2010;76:3886-97.

27. Edgar RC. UPARSE: highly accurate OTU sequences from microbial amplicon reads. Nat Methods. 2013;10(10):996-8.

28. Nelson MC, Morrison HG, Benjamino J, Grim SL, Graf J. Analysis, optimization and verification of Illumina-generated $16 \mathrm{~S}$ rRNA gene amplicon surveys. PLoS One. 2014;9:e94249

29. Kunin V, Engelbrektson A, Ochman H, Hugenholtz P. Wrinkles in the rare biosphere: pyrosequencing errors can lead to artificial inflation of diversity estimates. Environ Microbiol. 2010;12:118-23.

30. Bedding RA, Akhurst RJ. Simple technique for detection of insect parasitic Rhabditid nematodes in soil. Nematologica. 1975;21:109-10.

31. Poinar GO Jr, Thomas GM. Significance of Achromobacter nematophilus Poinar and Thomas (Achromobacteraceae: Eubacteriales) in the development of the nematode, DD-136 (Neoaplectana sp. Steinernematidae). Parasitology. 1966;56:385-90.

32. Boemare N, Thaler JO, Lanois A. Simple bacteriological tests for phenotypic characterization of Xenorhabdus and Photorhabdus phase variants. Symbiosis. 1997:22:167-75.

33. Tailliez P, Pages S, Ginibre N, Boemare N. New insight into diversity in the genus Xenorhabdus, including the description of ten novel species. Int J Syst Evol Microbiol. 2006;56:2805-18.

34. Gaudriault S, Duchaud E, Lanois A, Canoy AS, Bourot S, DeRose R, Kunst F, Boemare N, Givaudan A. Whole-genome comparison between Photorhabdus strains to identify genomic regions involved in the specificity of nematode interaction. J Bacteriol. 2006;188:809-14.

35. Andrews S. FastQC: a quality control tool for high throughput sequence data. 2010. https://www.bioinformatics.babraham.ac.uk/projects/fastqc/

36. Magoc T, Salzberg SL. FLASH: fast length adjustment of short reads to improve genome assemblies. Bioinformatics. 2011;27:2957-63.

37. Markowitz VM, Chen IMA, Palaniappan K, Chu K, Szeto E, Grechkin Y, et al. IMG: the integrated microbial genomes database and comparative analysis system. Nucleic Acids Res. 2012;40:D115-22.

38. Escudie F, Auer L, Bernard M, Mariadassou M, Cauquil L, Vidal K, et al. FROGS: find, rapidly, OTUs with galaxy solution. Bioinformatics. 2018;34:1287-94.

39. Callahan BJ, McMurdie PJ, Rosen MJ, Han AW, Johnson AJA, Holmes SP. DADA2: High-resolution sample inference from Illumina amplicon data. Nature Methods. 2016;13:581(7):581-583.

40. Mahe F, Rognes T, Quince C, de Vargas C, Dunthorn M. Swarm: robust and fast clustering method for amplicon-based studies. PeerJ. 2014;2:e593.

41. Edgar RC, Haas BJ, Clemente JC, Quince C, Knight R. UCHIME improves sensitivity and speed of chimera detection. Bioinformatics. 2011;27:2194-200.

42. Rognes T, Flouri T, Nichols B, Quince C, Mahe F. VSEARCH: a versatile open source tool for metagenomics. PeerJ. 2016;4:e2584.

43. Cole JR, Chai B, Farris RJ, Wang Q, Kulam-Syed-Mohideen AS, McGarrell DM, et al. The ribosomal database project (RDP-II): introducing myRDP space and quality controlled public data. Nucleic Acids Res. 2007;35:D169-72.

44. McMurdie PJ, Holmes S. Phyloseq: an R package for reproducible interactive analysis and graphics of microbiome census data. PLoS One. 2013;8:e61217.

45. Dixon P. VEGAN, a package of $R$ functions for community ecology. J Vegetation Science. 2003;14:927-30.

46. Wickham H. ggplot2. Wiley Interdisciplinary Reviews-Computational Statistics. 2011;3:180-5.

47. Kumar S, Stecher G, Tamura K. MEGA7: molecular evolutionary genetics analysis version 7.0 for bigger datasets. Mol Biol Evol. 2016;33:1870-4.

\section{Publisher's Note}

Springer Nature remains neutral with regard to jurisdictional claims in published maps and institutional affiliations.

\section{Ready to submit your research? Choose BMC and benefit from}

- fast, convenient online submission

- thorough peer review by experienced researchers in your field

- rapid publication on acceptance

- support for research data, including large and complex data types

- gold Open Access which fosters wider collaboration and increased citations

- maximum visibility for your research: over $100 \mathrm{M}$ website views per year

At BMC, research is always in progress.

Learn more biomedcentral.com/submissions 